\title{
TOXICIDAD RETINIANA SECUNDARIA A TRATAMIENTO CON CLOROQUINA
}

\author{
RETINAL TOXICITY FOLLOWING CHLOROQUINE THERAPY
}

\author{
FERRERAS A ${ }^{1}$, PINILLA I ${ }^{1}$, ABECIA E $^{1}$, PAJARÍN AB ${ }^{2}$, HONRUBIA FM $^{1}$
}

\begin{abstract}
RESUMEN
Caso clínico: Se describen dos casos de retinopatía tóxica bilateral en dos mujeres tratadas con cloroquina durante varios años por un lupus eritematoso sistémico.

Discusión: La retinopatía tóxica por cloroquina es una enfermedad infrecuente, iatrogénica y dosis dependiente, pero a pesar de ser conocida desde hace años y de realizarse revisiones oftalmológicas periódicas, siguen apareciendo nuevos casos.
\end{abstract}

Palabras claves: Retinopatía, maculopatía, cloroquina, hidroxicloroquina, toxicidad.

\begin{abstract}
Case report: Two cases of bilateral ocular toxicity following chloroquine therapy in two women with systemic lupus erythematosus are presented.

Discussion: Retinal toxicity due to chloroquine is an uncommon iatrogenic disease. It is dose-dependent. New cases are still being described despite the application of ophthalmic screening techniques (Arch Soc Esp Oftalmol 2007; 82: 103-108).
\end{abstract}

Key words: Retinopathy, maculopathy, chloroquine, hydroxychloroquine, toxicity.

\section{CASO CLÍNICO}

La cloroquina y su derivado la hidroxicloroquina fueron inicialmente usadas en el tratamiento y profilaxis de la malaria. Posteriormente se ha difundido su uso como fármacos muy eficaces en el control de enfermedades del tejido conectivo, especialmente en la artritis reumatoide y en el lupus eritematoso sistémico (LES). Pueden producir una enfermedad iatrogénica dosis dependiente que afecta a la retina, y que fue descrita por primera vez por Hoobs en 1959.

\section{Caso 1}

Mujer de 50 años con LES en tratamiento con un comprimido diario de 0,25 gramos de difosfato de cloroquina, equivalente a $150 \mathrm{mg}$ de cloroquina base, desde hacía más de 10 años (dosis total de cloroquina: $700 \mathrm{~g}$ aproximadamente). En las revisiones anuales previas no se apreciaron alteraciones hasta la última visita, en la que presentó: mejor agudeza visual corregida $(\mathrm{AV})$ de 10/10 en el ojo derecho

Recibido: 11/4/06. Aceptado: 22/12/06.

Hospital Universitario Miguel Servet. Zaragoza. España.

1 Doctor en Medicina. Oftalmología.

2 Doctor en Medicina. Medicina de familia. Euroresidencias. Zaragoza. España.

Correspondencia:

Antonio Ferreras

Hospital Universitario Miguel Servet. Servicio de Oftalmología

C/. Isabel la Católica, 1-3

50009 Zaragoza

España

E-mail: aferreras@msn.com 
(OD) y de 10/10 en el ojo izquierdo (OI), biomicroscopía y tonometría normales. Se realizó una perimetría automatizada convencional (PA) que mostró una importante caída de los valores umbrales centrales (fig. 1) en ambos ojos (AO). El test de colores Ishihara fue normal. En el fondo de ojo se observaron cambios pigmentarios a nivel macular inespecíficos (fig. 2). Se suspendió el tratamiento con cloroquina y se realizaron revisiones oftalmológicas, en las que no se apreciaron cambios en la AV ni en el campo visual.

\section{Caso 2}

Mujer de 40 años en tratamiento con un comprimido diario de 0,25 gramos de difosfato de cloroquina por LES, de forma casi continuada desde hacía 9 años (dosis total de cloroquina: aproximadamente 293 g). Había sido revisada en otro centro sin haber presentado ninguna alteración. Acudió refiriendo dificultad para enfocar y diferenciar los colores desde hacía 2 meses. La AV era de 1 en AO, biomicroscopía y tonometría normales. En la PA se
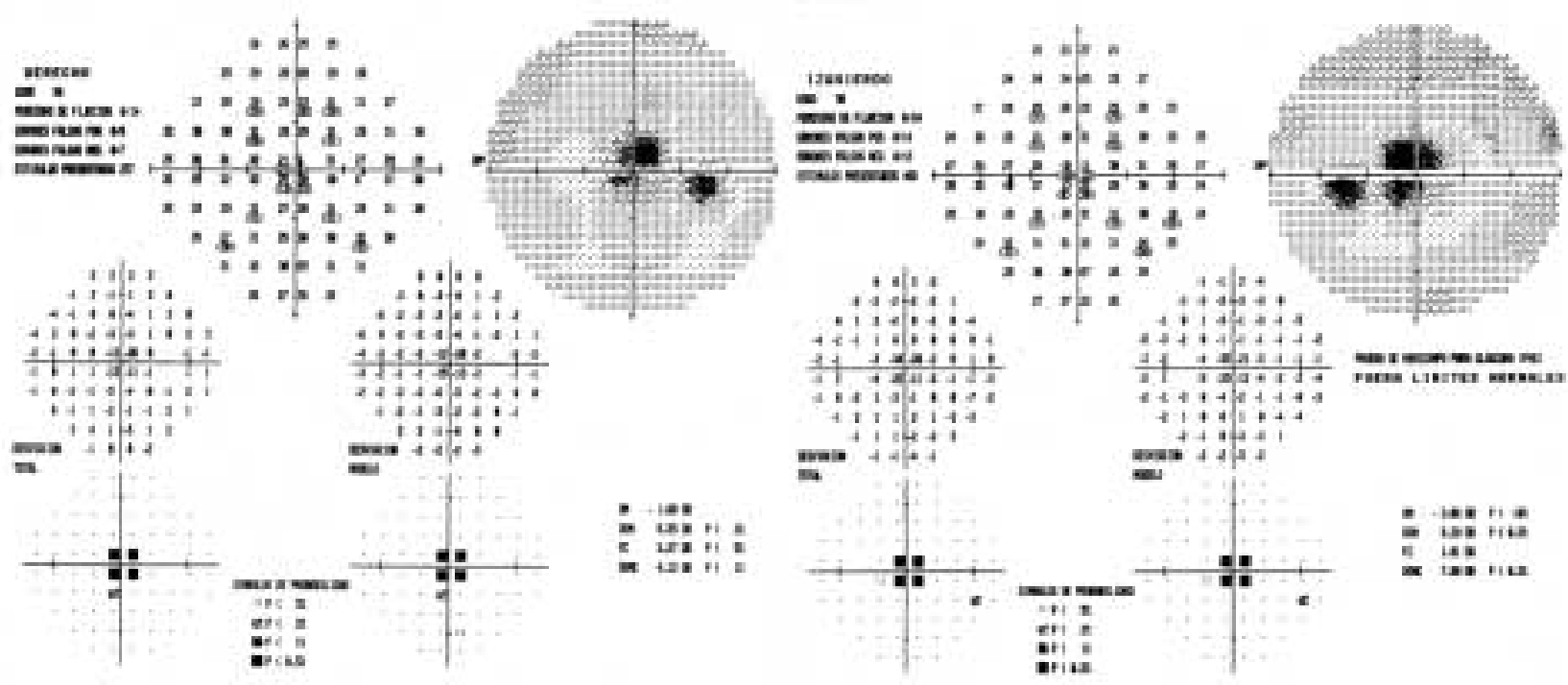

Fig. 1: Perimetría (Humphrey 30-2 umbral completo) del primer caso clínico, que muestra el defecto a nivel central.

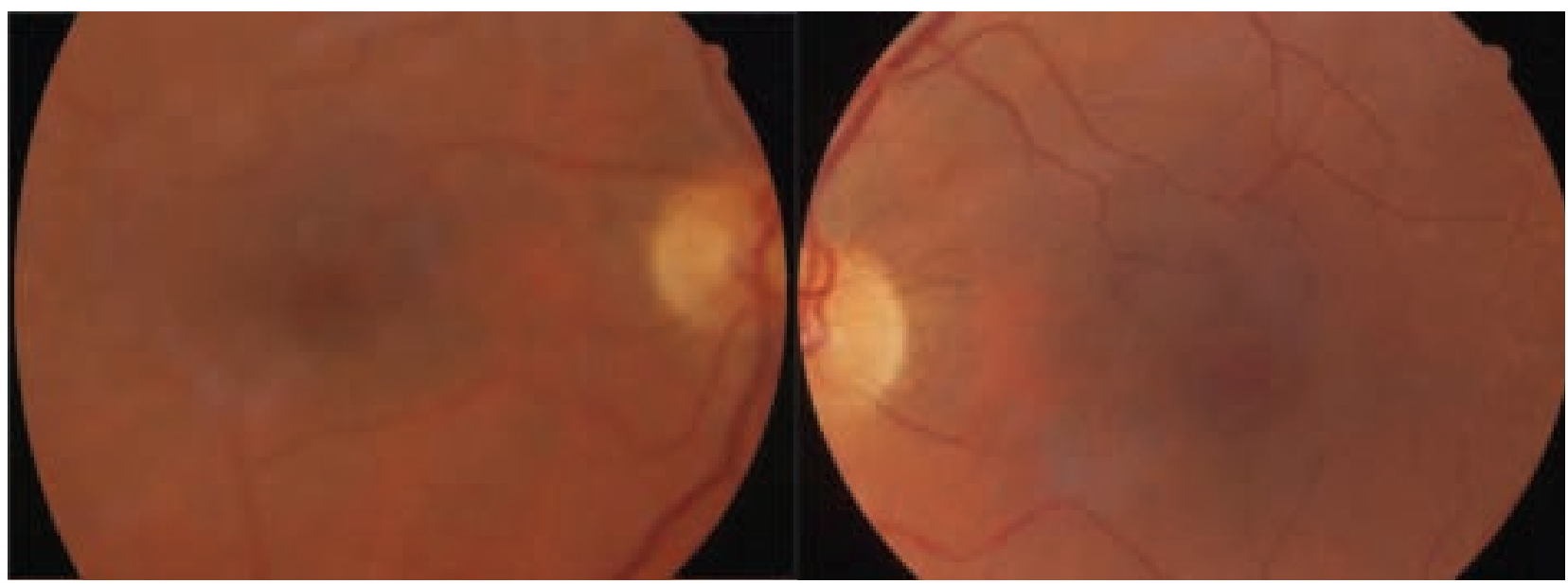

Fig. 2: Aspecto del fondo de ojo del caso clínico 1. Presentaba cambios pigmentarios inespecíficos. 
observó una reducción de los valores de umbral centrales y paracentrales en $\mathrm{AO}$ (fig. 3). En el Ishihara (15 láminas) presentó 6 fallos en el OD y 5 fallos en el OI. El aspecto del fondo de ojo presentaba la característica imagen de hiperpigmentación en ojo de buey (fig. 4). Se retiró el tratamiento con cloroquina pero siguió empeorando. A los dos meses la AV fue de 7/10 para el OD y 8/10 para el OI. Se realizó una perimetría de duplicación de fre- cuencia que presentó valores umbral de $0 \mathrm{~dB}$ a nivel central (fig. 5). A los 6 meses la AV bajó a 4/10 en AO. Se repitió la PA con la estrategia 10-2 (fig. 6) que mostró con más detalle el defecto del campo visual central. Los potenciales visuales evocados y el electrorretinograma fueron normales; sin embargo, el electrooculograma presentó valores disminuidos. El aspecto funduscópico fue similar. En las revisiones siguientes no se observaron cambios.
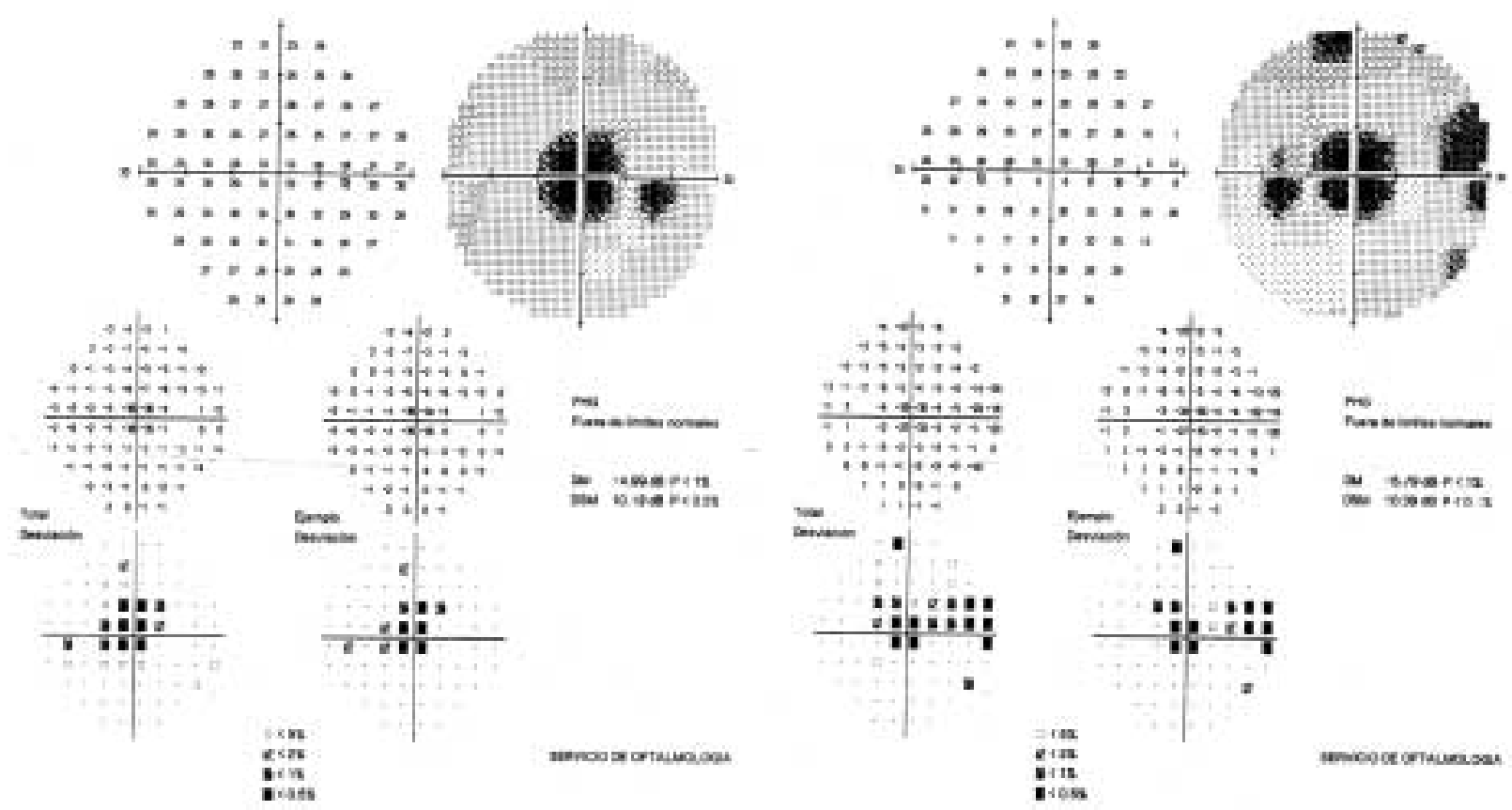

Fig. 3: Perimetría (Humphrey 30-2 SITA Standard) del segundo caso clínico, que muestra el defecto a nivel central que se extiende hacia el área temporal en el ojo izquierdo.

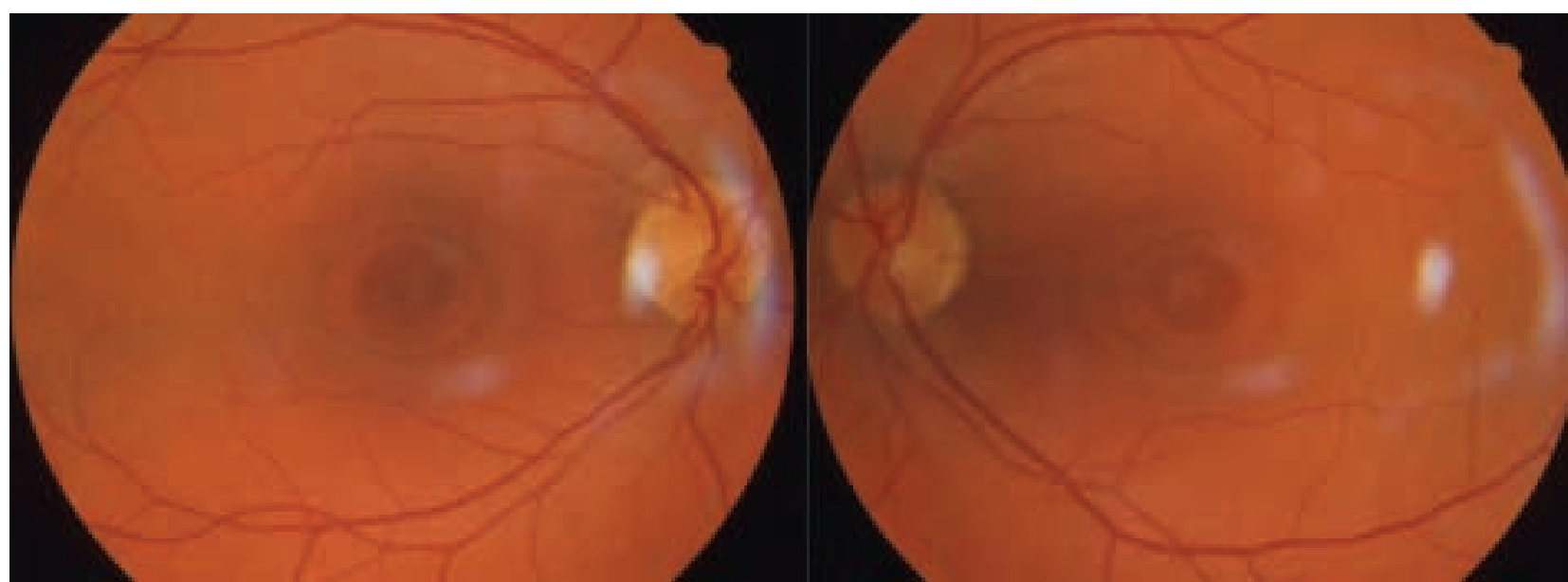

Fig. 4: Aspecto del fondo de ojo del caso clínico 2. Presentaba la clásica imagen de maculopatía en ojo de buey. 
LEFT EYE

Test Duration: $\quad 4: 58$
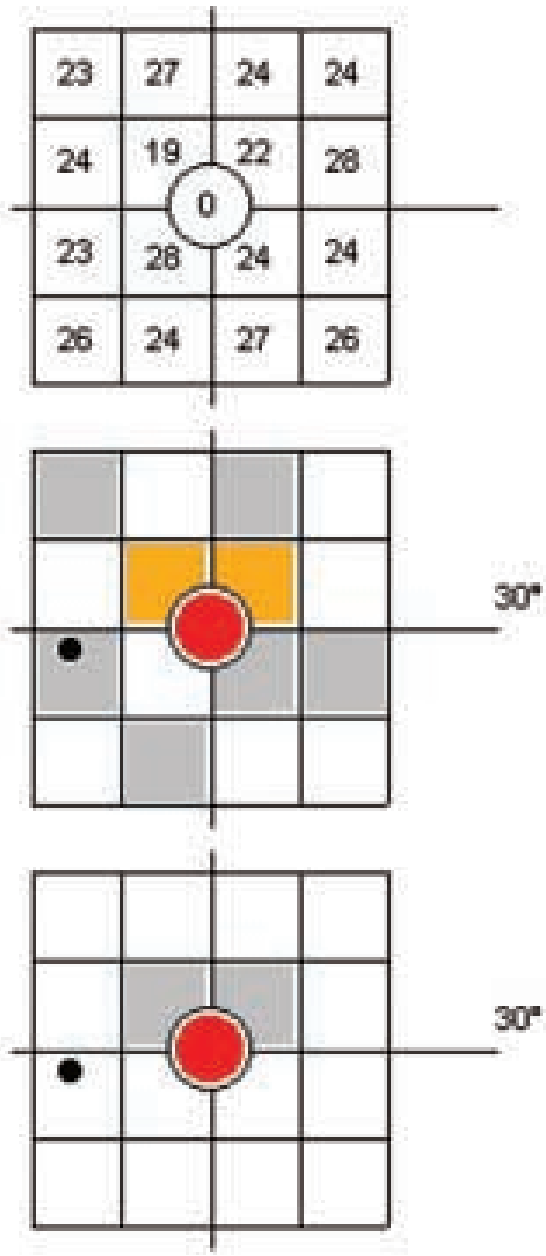

MD: $\quad-5.60 \mathrm{~dB} P<0.5 \%$

PSD: $\quad+7.92 \mathrm{dA} P<2 \%$

FIXATION ERRS:

FALSE POS ERTS

FALSE NEG ERRS

$0 / 6$

$0 / 6$

$0 / 3$
Threshold (dB)

Total Deviation

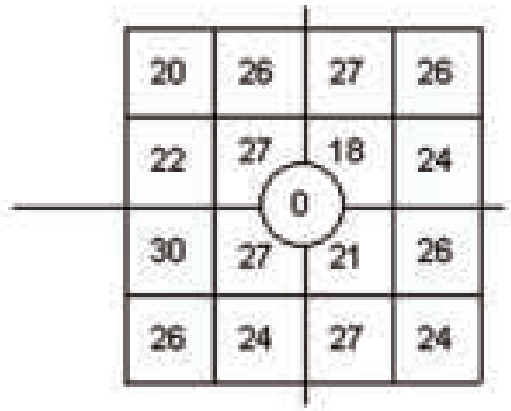

$30^{*}$

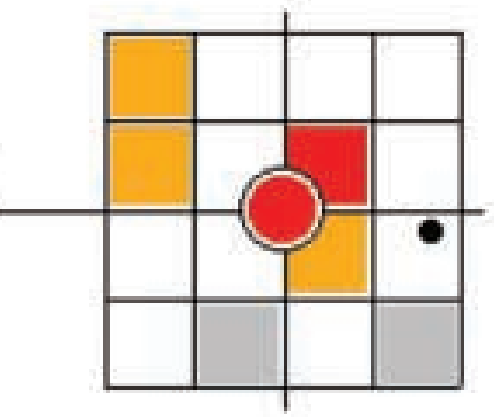

Pattern Deviation

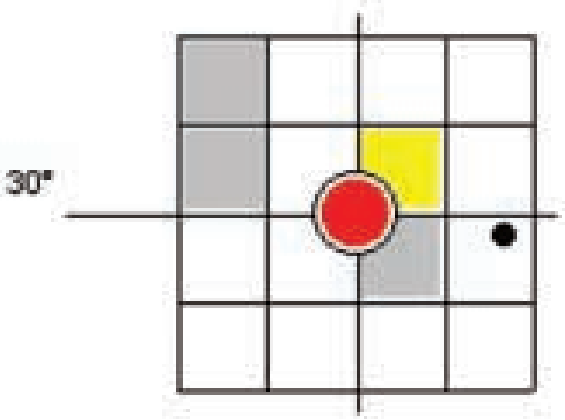

MD: $\quad-5.68 d \theta \quad P<0.5 \%$

PSO $\quad+8.84 d \theta \quad P<1 \%$

FIXATIONERRS: $\quad 0 / 6$

FALSE POS ERRS: $0 / 6$

FALSE NEG ERRS: $\quad 0 / 3$

Fig. 5: Perimetría de duplicación de frecuencia (estrategia C-20 umbral completo) del segundo caso clínico, que muestra valores umbral de $0 \mathrm{~dB}$ a nivel central.

\section{DISCUSIÓN}

Los mecanismos por los que se produce la retinopatía tóxica no son bien conocidos aunque pare- ce que el estrés oxidativo que ocasionan los antipalúdicos a nivel de la retina jugaría un papel importante (1). También se ha visto que las mutaciones del gen ABCR (también conocido como ABCA4), 

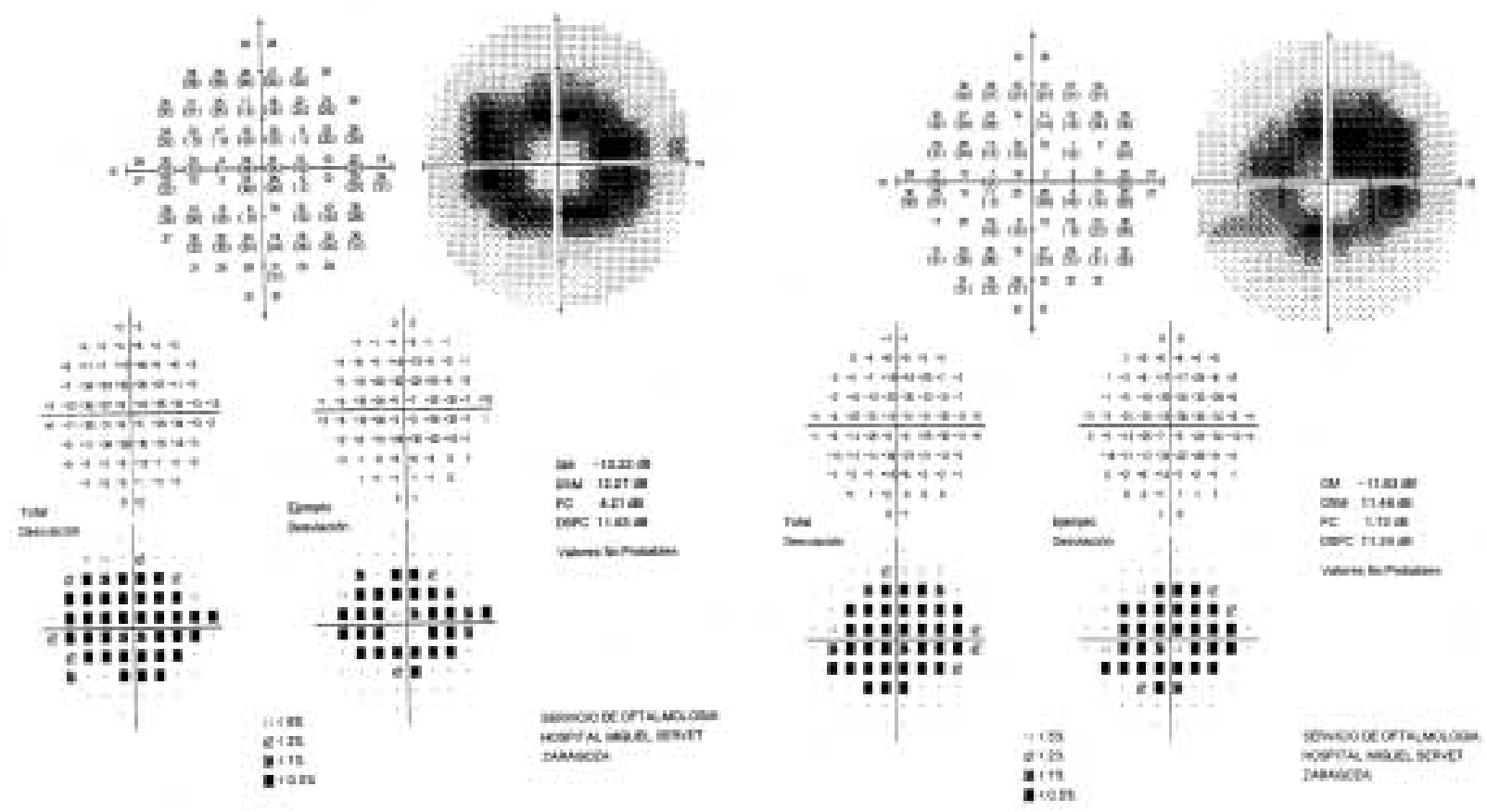

Fig. 6: Perimetría (Humphrey 10-2 umbral completo) del segundo caso clínico a los 6 meses, que muestra con detalle la afectación del campo visual central.

relacionado con la enfermedad de Stargardt y con la degeneración macular asociada a la edad, predisponen a desarrollar la retinopatía tóxica (2).

La realización de pruebas de despistaje para la retinopatía por cloroquina ha sido muy discutida. Los últimos estudios $(3,4)$ coinciden en que no es necesario realizar pruebas de cribaje cuando las dosis de tratamiento son bajas $(3 \mathrm{mg} / \mathrm{kg} /$ día para cloroquina y $6,5 \mathrm{mg} / \mathrm{kg} /$ día para hidroxicloroquina). En estos casos, sería suficiente efectuar los exámenes oftalmológicos recomendados para cualquier persona y cuya frecuencia depende de la edad. El despistaje estaría indicado sólo en casos de alto riesgo, que vendrá determinado por el tipo de fármaco, la dosis empleada, la duración del tratamiento superior a 5 años, el peso, la edad, la función renal y la presencia o no de enfermedades retinianas concomitantes (3). Se recomienda una exploración oftalmológica completa y un campo visual central tanto en la primera visita como en las siguientes (3). Como pruebas opcionales se han sugerido los test de colores, la rejilla de Amsler, las fotografías del fondo de ojo y pruebas electrofisiológicas.

Las pruebas electrofisiológicas tienen una validez relativa. El electrorretinograma multifocal parece ser más adecuado para evaluar la toxicidad reti- niana por cloroquina. Puede presentar una pérdida de las respuestas centrales y paracentrales mientras que el electrorretinograma de campo total puede permanecer normal $(3,5)$. La angiografía con fluoresceína ayuda poco al diagnóstico de la retinopatía pero puede ser útil para descartar otras patologías concomitantes.

Ningún tratamiento ha demostrado ser eficaz una vez que se produce el efecto tóxico, por lo que la única actitud a seguir es suspender la administración del fármaco.

La retinopatía puede avanzar a pesar de suspender el tratamiento. Un fondo de ojo completamente normal no excluye la enfermedad. Siguen apareciendo casos a pesar de conocerse la toxicidad y hacerse seguimientos oftalmológicos.

\section{BIBLIOGRAFÍA}

1. Toler SM. Oxidative stress plays an important role in the pathogenesis of drug-induced retinopathy. Exp Biol Med 2004; 229: 607-615.

2. Shroyer NF, Lewis RA, Lupski JR. Analysis of the ABCR $(A B C A 4)$ gene in 4-aminoquinoline retinopathy: Is retinal toxicity by chloroquine and hydroxychloroquine related to Stargardt disease? Am J Ophthalmol 2001; 131: 761-766. 
3. Marmor MF, Carr RE, Easterbrook M, Farjo AA, Mieler WF; American Academy of Ophthalmology. Recommendations on screening for chloroquine and hydroxychloroquine retinopathy: a report by the American Academy of Ophthalmology. Ophthalmology 2002; 109: 1377-1382.

4. Grierson DJ. Hydroxychloroquine and visual screening in a rheumatology outpatient clinic. Ann Rheum Dis 1997; 56: $188-190$

5. Neubauer AS, Samari-Kermani $K$, Schaller $U$, WelgeUbetaen U, Rudolph G, Berninger T. Detecting chloroquine retinopathy: electro-oculogram versus colour vision. Br J Ophthalmol 2003; 87: 902-908. 